\title{
Incorporation of optically active erbium into GaAs using the novel precursor tris(3,5-di-tert-butylpyrazolato)bis(4-tert-butylpyridine)erbium
}

\author{
J. G. Cederberg, ${ }^{\text {a) }}$ T. D. Culp, and B. Bieg ${ }^{\text {) }}$ \\ Department of Chemical Engineering, University of Wisconsin-Madison, 1415 Engineering Drive, Madison, \\ Wisconsin 53706 \\ D. Pfeiffer and C. H. Winter \\ Department of Chemistry, Wayne State University, Detroit, Michigan 48202 \\ K. L. Bray \\ Department of Chemistry, Washington State University, Pullman, Washington 99164 \\ T. F. Kuech \\ Department of Chemical Engineering, University of Wisconsin-Madison, 1415 Engineering Drive, Madison, \\ Wisconsin 53706
}

(Received 30 December 1997; accepted for publication 2 November 1998)

\begin{abstract}
We have investigated the use of an alternative erbium precursor, tris(3,5-di-tert-butylpyrazolato)bis(4-tert-butylpyridine)erbium, to dope erbium into GaAs. The incorporated erbium forms an optically active center identified as $\mathrm{Er}-2 \mathrm{O}$. The GaAs: $\mathrm{Er}$ formed using this precursor exhibits sharper and more intense optical emission, attributed to the $\mathrm{Er}-2 \mathrm{O}$ center, than that previously found with cylcopentadienyl-based erbium sources. Codoping GaAs:Er with shallow donors results in a quenching of the erbium-related luminescence, while codoping with shallow acceptors results in no significant change in the spectrum. Mechanisms for the observed luminescence-quenching behavior are discussed. Deep level transient spectroscopy performed on silicon or selenium codoped GaAs:Er showed the presence of several electron traps in the upper half of the band gap. The origins of these electron traps are considered. (C) 1999 American Institute of Physics. [S0021-8979(99)10903-4]
\end{abstract}

\section{INTRODUCTION}

Semiconductors doped with rare-earth impurities are the focus of scientific and technological interest. Rare-earth isoelectronic impurities incorporated into semiconductors would allow the integration of optical and electronic components through the development of temperature invariant light sources in the near infrared (IR) spectral range. The incorporation of erbium into silicon has been investigated for such optoelectronic applications. ${ }^{1,2}$ Compound semiconductors have also been investigated as rare-earth ion hosts and have several advantages over silicon-based approaches that include a reduction of the thermal quenching of the luminescent lifetime and intensity. ${ }^{3}$

Erbium has been incorporated into GaAs during crystal growth and through postgrowth processing. Molecular beam epitaxy (MBE) ${ }^{4,5}$ ion implantation, ${ }^{6}$ and liquid phase epitaxy $(\mathrm{LPE})^{7}$ have been used to synthesize erbium-doped GaAs. These methods rely on the use of elemental erbium, which is difficult to maintain in a pure state due to its high reactivity. Metalorganic vapor phase epitaxy (MOVPE) can be used to grow GaAs:Er, but it requires a metalorganic erbium source with sufficient vapor pressure. ${ }^{8}$ Chemical precursors for erbium can offer advantages over elemental erbium since such

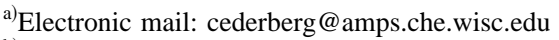

${ }^{b)}$ On leave from Szczecin Maritime University, Waly Chrobrego 1/2, Szczecin, Poland.
}

sources can be purified and thus may have a reduced risk of source contamination. Several erbium compounds have been previously investigated for use in MOVPE growth. Cyclopentadienyl (Cp)-based erbium source have been most commonly used due to their ease of synthesis and their similarity to bis-cyclopentadienyl magnesium. ${ }^{9,10} \mathrm{Cp}$-based sources are often employed as volatile precursors for transition metals and lanthanide elements. Cp-based erbium sources have been shown, however, to coincorporate carbon into the GaAs films ${ }^{11}$ that could alter the microscopic environment of the erbium center. ${ }^{12}$ Sources based on amide ligand chemistries have also been investigated, but these source compounds can contain other potential impurities, such as silicon. ${ }^{13}$ Cointroduction of other unintentional impurities can result in the formation of undesirable erbium complexes and produce an $n$ - or $p$-type background. An erbium precursor that is free of such "unintentional" and undesired impurities is presented in this study. We have investigated the use of an alternative erbium precursor, tris(3,5-di-tert-butylpyrazolato)bis(4-tert-butylpyridine)erbium, abbreviated $\left(t-\mathrm{Bu}_{2} \mathrm{Pz}\right)_{3}(4-t-\mathrm{BuPy})_{2} \mathrm{Er}$, where $\mathrm{Pz}$ denotes a pyrazolato ligand and $\mathrm{Py}$ denotes a pyridine ligand that contains no direct $\mathrm{Er}-\mathrm{C}$ or $\mathrm{Er}-\mathrm{Si}$ bonds. In this article, a comprehensive study of the electrical and optical properties of GaAs films doped with erbium using this new precursor is presented. The effect of shallow impurities on the erbium-related luminescence is reported and several mechanisms for erbium- 
related luminescence quenching are discussed. The change in the deep level structure of GaAs as erbium is added is also reported. The origin of these erbium-related deep levels is considered.

\section{EXPERIMENT}

$\left(t-\mathrm{Bu}_{2} \mathrm{Pz}\right)_{3}(4-t-\mathrm{BuPy})_{2} \mathrm{Er}$ is a solid source at room temperature with a melting point of $241{ }^{\circ} \mathrm{C}$ and a sublimation point of $150{ }^{\circ} \mathrm{C}$ at 0.1 Torr. During sample growth, the source bubbler was heated to approximately $90{ }^{\circ} \mathrm{C}$ with downstream lines maintained at a higher temperature to avoid source condensation. The synthesis of $\left(t-\mathrm{Bu}_{2} \mathrm{Pz}\right)_{3}(4-t-\mathrm{BuPy})_{2} \mathrm{Er}$ has been described in detail elsewhere. ${ }^{14}$

Samples of GaAs and GaAs:Er were grown in a horizontal MOVPE reactor at 78 Torr using hydrogen purified by a palladium cell as the carrier gas. Trimethylgallium (TMGa) and $\mathrm{AsH}_{3}$ were used as the growth precursors. All growths were carried out on (100) GaAs substrates misoriented $2^{\circ}$ from (100) toward (110). The films doped with erbium were observed to have a hazy appearance after growth, whereas the undoped GaAs had a specular surface. Several dopants were used for $n$ - and $p$-type doping. Disilane $\left(\mathrm{Si}_{2} \mathrm{H}_{6}\right)$, diluted to $10 \mathrm{ppm}$ in $\mathrm{H}_{2}$, and hydrogen selenide $\left(\mathrm{H}_{2} \mathrm{Se}\right)$, diluted to 30 ppm in $\mathrm{H}_{2}$, were used as the shallow donor sources. Dimethyl zinc (DMZn), diluted to $45 \mathrm{ppm}$ in $\mathrm{H}_{2}$, was used for $p$-type doping. This combination of shallow impurities $(\mathrm{Si}$, $\mathrm{Se}$, and $\mathrm{Zn}$ ) allowed us to determine the effect of material conductivity type on the erbium-related luminescence and to evaluate the possibility of complex formation that could affect the erbium-related luminescence.

The incorporation of erbium from $\left(t-\mathrm{Bu}_{2} \mathrm{Pz}\right)_{3}(4-t-\mathrm{BuPy})_{2} \mathrm{Er}$ was controlled by altering the flow rate of the source compound during growth. The specific dopant mole fraction could not be determined since the vapor pressure of this compound at the source temperature used is not known. The amount of electrical compensation related to the presence of erbium in the reactor during crystal growth was determined by electrochemical capacitancevoltage $(E C-V)$ profiling. The effect of varying growth parameters on the amount of compensation was evaluated using $E C-V$ profiling of multilayer structures. In these experiments, the $\mathrm{H}_{2}$ flux through the erbium source bubbler was maintained at $200 \mathrm{sccm}$ in a total reactor flow of 7.5 slpm. The film growth temperature was varied from 600 to $750{ }^{\circ} \mathrm{C}$ in a stepwise fashion during growth, while maintaining a constant anion-to-cation ratio (V/III). The effect of the V/III was studied by a similar growth structure. The V/III ratio values of 30,60 , and 90 were used at a growth temperature of $600{ }^{\circ} \mathrm{C}$. The samples for the optical and electrical studies consisted of a single layer of GaAs, nominally $3 \mu \mathrm{m}$ thick, grown on top of an undoped GaAs buffer on a GaAs substrate. Secondary ion mass spectroscopy (SIMS) measurements were performed on selected samples in an attempt to quantify the erbium and oxygen contents of the films. The deep level structure was determined by deep level transient spectroscopy (DLTS) and capacitance-voltage $(C-V)$ measurements on silicon or selenium codoped GaAs:Er samples.

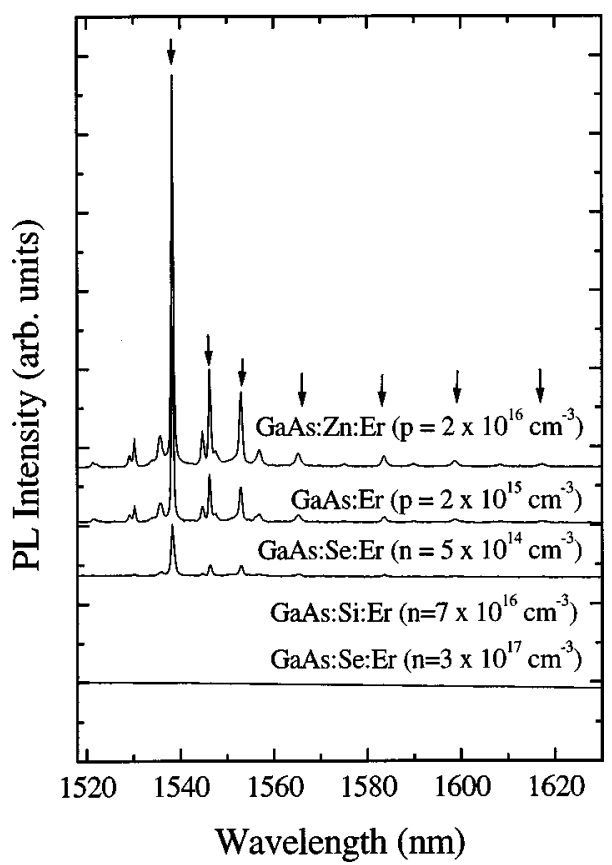

FIG. 1. Photoluminescence spectra taken at $12 \mathrm{~K}$ for GaAs doped only with erbium and codoped with zinc, selenium, and silicon. The PL intensity is decreased as the Fermi level approaches the conduction band. The vertical arrows mark peaks in the spectrum previously assigned to the $\mathrm{Er}-2 \mathrm{O}$ center (after Ref. 28). The additional lines are identified with other transitions from the ${ }^{4} I_{13 / 2}$ manifold. The samples were grown at $600{ }^{\circ} \mathrm{C}$ with a V/III of 60 .

Schottky diodes were fabricated for DLTS measurement using a $\mathrm{Cr} / \mathrm{Au}(20 / 150 \mathrm{~nm})$ multilayer. A backside ohmic contact was made to the $n$-type substrate by using an alloyed AuGe (12\%)/Ni/Ti/Au (80/20/20/80 nm) multilayer. The use of a Schottky diode in the DLTS measurements limits the range of detectable emission energies to about half of the band gap. DLTS measurements were not possible on the GaAs:Zn-based samples due to the large leakage currents found in the $p$-type Schottky structures. For the photoluminescence (PL) measurements, all samples were cooled to 12 $\mathrm{K}$ and the emission was excited by the $514 \mathrm{~nm}$ line of an argon ion laser. The signal was dispersed by a single grating monochromator and detected with a liquid nitrogen cooled germanium detector. All samples were measured under the same conditions of temperature, excitation power density and wavelength, instrument geometry, and resolution.

\section{RESULTS}

PL measurements of GaAs doped with only erbium yielded the spectrum shown in Fig. 1. The features marked with arrows have previously been assigned to the $\mathrm{Er}-2 \mathrm{O}$ center. ${ }^{15}$ The remaining lines are due to transitions from higher energy levels in the ${ }^{4} I_{13 / 2}$ manifold that are occupied due to laser heating. Codoping with silicon or selenium leads to quenching of the erbium-related luminescence, also shown in Fig. 1. To further investigate the effect of shallow donors on the luminescence, GaAs:Se:Er having an electron concentration of only $n \sim 5 \times 10^{14} \mathrm{~cm}^{-3}$ was grown. The intensity of the erbium-related luminescence is reduced, but not completely quenched. Codoping with zinc, however, leads to a 


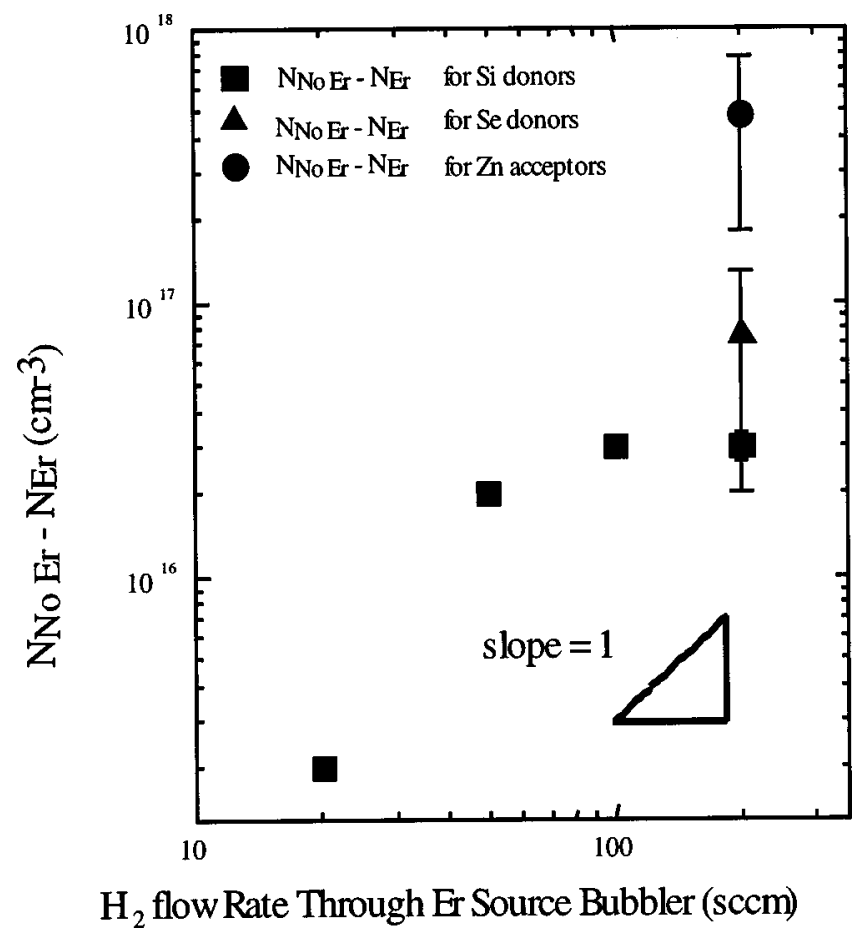

FIG. 2. Compensation of shallow donors and acceptors by erbium-related levels as a function of $\mathrm{H}_{2}$ flow through the erbium source bubbler is noted by the difference in the carrier concentration between the Er-doped and non-Er doped samples at a constant concentration of shallow dopants. The background shallow donor or acceptor concentration is kept constant at 1 $\times 10^{17} \mathrm{~cm}^{-3}$ for silicon. For selenium and zinc the background donor concentration varied over the range of $5 \times 10^{17}-3.5 \times 10^{17} \mathrm{~cm}^{-3}$ for selenium and $8 \times 10^{17}-2 \times 10^{17} \mathrm{~cm}^{-3}$ for zinc. A higher level of carrier compensation was observed in $p$-type material. For all cases, the growth temperatures were $600{ }^{\circ} \mathrm{C}$ and the V/III ratios were 60 .

twofold increase in the magnitude of the erbium-related luminescence as is noted in Fig. 1 under identical measurement conditions.

The electrical compensation, $N_{\mathrm{No} \text { Er }}-N_{\mathrm{Er}}$, where $N$ is the carrier concentration, measured by $E C-V$ in GaAs samples doped with silicon, selenium, or zinc, with or without erbium, is shown in Fig. 2 as a function of the $\mathrm{H}_{2}$ flow rate through the erbium source bubbler (related to the erbium mole fraction in the reactor). This definition of compensation is a direct measure of the loss of carriers due to the introduction of the erbium source into the reactor. In silicon-doped GaAs:Er, the electrically active concentration appears to be slightly sublinear with the compensation saturating at high $\mathrm{H}_{2}$ (Er) flow rates. In zinc-doped GaAs:Er, a larger degree of compensation was observed for a similar erbium source flow rate. The measured compensation, at a given erbium flow rate, for $n$-type samples appears to be independent of the donor species within our measurement uncertainty. The amount of compensation in $p$-type GaAs is almost one order of magnitude greater than that in $n$-type GaAs for the same gas phase erbium concentration. SIMS could not detect measurable amounts of erbium, indicating that the concentration was below the SIMS detection limit, which was 2 $\times 10^{15} \mathrm{~cm}^{-3}$. SIMS analysis indicated that the GaAs:Er samples possessed an oxygen content of $2 \times 10^{17} \mathrm{~cm}^{-3}$ in the epilayer. The carbon present in the erbium-doped layers
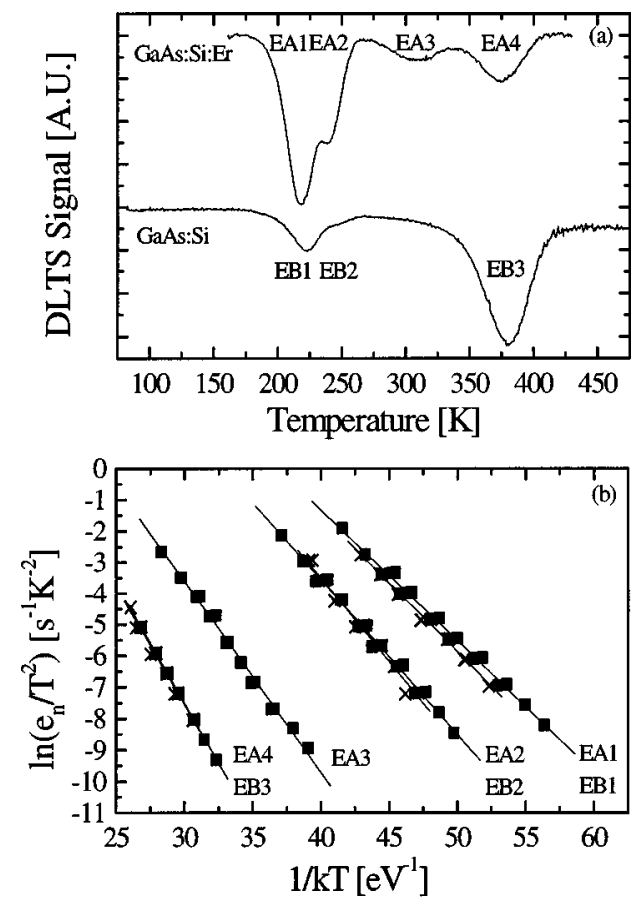

FIG. 3. (a) DLTS spectra for GaAs:Si and GaAs:Si:Er. (b) Arrhenius plots for the levels detected in GaAs:Si $(\times)$ and GaAs:Si:Er ( $\mathbf{\square})$. Only level EA3 is directly attributed to erbium incorporation. Level $E A 4$ is attributed to the $\operatorname{As}_{\mathrm{Ga}}$ (EL2) (after Ref. 17) defect while EA1 and EA2 are believed to be due to unidentified contamination in the erbium source.

varied from $1.5 \times 10^{17}$ to $7 \times 10^{16} \mathrm{~cm}^{-3}$ depending on the specific growth conditions. The oxygen and carbon levels in the nonerbium-doped samples have previously been found to be below SIMS detection limits of $7 \times 10^{16} \mathrm{~cm}^{-3}$ for oxygen and $1 \times 10^{16}$ for carbon. No oxygen-related DLTS peaks were found in the $n$-type control samples that contained no erbium.

The electrical compensation associated with the erbium incorporation, based on $E C-V$ measurements, was reduced at increased growth temperatures. The degree of electrical compensation decreases with an increased V/III ratio by approximately a factor of 2 as the V/III increased from 30 to 90. Higher V/III ratios can lead to reduced unintentional carbon incorporation and a slight increase in the concentration of silicon donors from $\mathrm{Si}_{2} \mathrm{H}_{6}$. These factors make determination of minor changes in compensation due to erbium-related effects difficult to separate from those associated with changes in the growth temperature and V/III ratio.

DLTS measurements were taken in a $80-450 \mathrm{~K}$ temperature range for the GaAs:Si:Er and GaAs:Se:Er samples and their respective control samples were doped only with shallow impurities. For GaAs:Si:Er four deep levels, labeled $E A 1-E A 4$, were detected in the spectrum shown in Fig. 3(a). In GaAs:Si control sample traps $E B 1-E B 3$ were observed. The Arrhenius plots for the levels detected are shown in Fig. 3(b) and the trap energies, capture cross sections, and concentrations measured are tabulated in Table I. The concentration of the trap $E B 1$ in GaAs:Si is approximately half that of $E A 1$ observed in GaAs:Si:Er, while the concentrations of $E A 2$ and $E B 2$ are roughly the same. The similarity 
TABLE I. Experimentally determined trap characteristics obtained for GaAs:Si and GaAs:Si:Er. The emission energy reported for level $E C 1$ represents zero-field energy.

\begin{tabular}{lcccc}
\hline \hline Sample & Trap & $\begin{array}{c}\text { Activation } \\
\text { energy } \\
(\mathrm{eV})\end{array}$ & $\begin{array}{c}\text { Capture cross } \\
\text { section } \\
\left(\mathrm{cm}^{2}\right) \times 10^{14}\end{array}$ & $\begin{array}{c}\text { Trap } \\
\text { concentration } \\
\left(\mathrm{cm}^{-3}\right) \times 10^{-14}\end{array}$ \\
\hline GaAs:Si & $E B 1$ & $0.44 \pm 0.01$ & $4.2 \pm 2.4$ & $5.8 \pm 0.5$ \\
& $E B 2$ & $0.53 \pm 0.02$ & $26.5 \pm 21.9$ & $1.6 \pm 0.5$ \\
& $E B 3$ & $0.75 \pm 0.02$ & $1.6 \pm 0.8$ & $17.1 \pm 0.5$ \\
GaAs:Si:Er & $E A 1$ & $0.42 \pm 0.01$ & $2.0 \pm 0.9$ & $10.6 \pm 0.5$ \\
& $E A 2$ & $0.49 \pm 0.01$ & $4.3 \pm 2.3$ & $1.5 \pm 0.5$ \\
& $E A 3$ & $0.77 \pm 0.04$ & $0.9 \pm 0.3$ & $1.3 \pm 0.5$ \\
& $E A 4$ & $0.76 \pm 0.03$ & $1.8 \pm 0.3$ & $3.9 \pm 0.5$ \\
\hline \hline
\end{tabular}

in emission energy and capture cross section of $E A 1$ and $E B 1$ indicates a common, unidentified origin for these levels. A level at $0.44 \mathrm{eV}$ has been previously detected in undoped, $n$-type GaAs grown by MOVPE. ${ }^{16}$ Traps EA4 and $E B 3$ have similar emission energies and capture cross sections, however, the concentration observed in GaAs:Si is over four times that observed in GaAs:Si:Er. The emission energies and capture cross sections of $E A 4$ and $E B 3$ are very close to published data for the $E L 2\left(\mathrm{As}_{\mathrm{Ga}}\right)$ defect. ${ }^{17}$ The only level that is not observed in the control sample is $E A 3$, which indicates this peak is directly or indirectly related to the presence of erbium. For GaAs:Si:Er the temperature positions of peaks $E A 1, E A 2$, and $E A 4$ were independent of the amplitude and of the duration of the filling pulse. The temperature position of level EA3 was dependent on the height of the filling pulse, indicating an electric field dependence for this trap. The variation in the emission rate with electric field is shown in Fig. 4 for three different measurement temperatures. At constant temperature, the emission rate $e_{n}$, follows the relationship:

$$
e_{n}=e_{n 0} \exp \left[\alpha_{\mathrm{FP}} F^{1 / 2} / k T\right],
$$

where $F$ is the electric field, $e_{n 0}$ is the zero field emission rate, $k$ is the Boltzmann constant, and $T$ is the absolute temperature. A Frenkel-Poole constant of $\alpha_{\mathrm{FP}}=2.7$ $\times 10^{-4}\left(\mathrm{eV}^{2} \mathrm{~cm} / \mathrm{V}\right)^{1 / 2}$ was determined for all measurement temperatures. A constant value of $\alpha_{\mathrm{FP}}$ at different temperatures combined with an emission rate that varies linearly with the square root of the electric field strongly indicates the presence of Frenkel-Poole barrier reduction. ${ }^{18}$ The value of

TABLE II. Experimentally determined trap characteristics obtained for GaAs:Se and GaAs:Se:Er. The trap concentrations for $H D 1$ could not be determined because electronic saturation of this level was not observed for the testing conditions available.

\begin{tabular}{lcccc}
\hline \hline Sample & Trap & $\begin{array}{c}\text { Activation } \\
\text { energy } \\
(\mathrm{eV})\end{array}$ & $\begin{array}{c}\text { Capture cross } \\
\text { section } \\
\left(\mathrm{cm}^{2}\right) \times 10^{14}\end{array}$ & $\begin{array}{c}\text { Trap } \\
\text { concentration } \\
\left(\mathrm{cm}^{-3}\right) \times 10^{-14}\end{array}$ \\
\hline GaAs:Se & $H D 1$ & $0.61 \pm 0.02$ & $2.0 \pm 1.4$ & $\ldots$ \\
& $E D 2$ & $0.72 \pm 0.02$ & $0.5 \pm 0.2$ & $230 \pm 5$ \\
GaAs:Se:Er & $E C 1$ & $0.47 \pm 0.01$ & $0.09 \pm 0.02$ & $23 \pm 0.5$ \\
& $E C 2$ & $0.69 \pm 0.01$ & $0.5 \pm 0.2$ & $27 \pm 0.5$ \\
\hline \hline
\end{tabular}

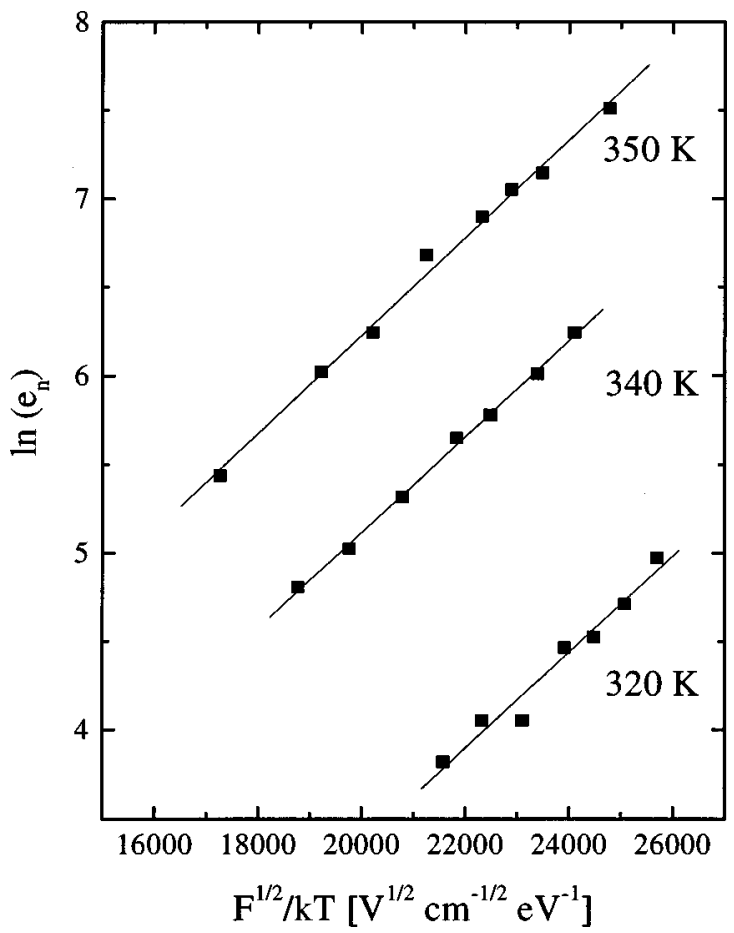

FIG. 4. Effect of internal electric field on trap EA3. This trap exhibits Frenkel-Poole barrier lowering as evidenced by the value of $\alpha_{\mathrm{PF}}$ and the linear dependence of $\ln \left(e_{n}\right)$ on $F^{1 / 2}$.

$\alpha_{\mathrm{PF}}$ calculated from Fig. 4 is only slightly smaller than the theoretical value for GaAs of $4 \times 10^{-4}\left(\mathrm{eV}^{2} \mathrm{~cm} / \mathrm{V}\right)^{1 / 2} \cdot{ }^{19} \mathrm{~A}$ zero field activation energy of $0.77 \mathrm{eV}$ was obtained by extrapolating the emission rate to zero field.

The DLTS spectra of GaAs:Se:Er, shown in Fig. 5(a), shows the presence of two levels, $E C 1$ and $E C 2$, in contrast to GaAs:Se, which contained a high concentration of $E D 2$ and an anomalous hole trap $H D 1$. The parameters of the levels detected in the GaAs:Se and GaAs:Se:Er samples are tabulated in Table II. The hole trap has been observed previously in $\mathrm{Al}_{0.22} \mathrm{Ga}_{0.78} \mathrm{As}: \mathrm{Se}$ and was attributed to Se concentrations greater than $2 \times 10^{17} \mathrm{~cm}^{-3}$. ${ }^{20}$ Since the hole trap is also present in the GaAs:Se:Er, the emission energies for $E C 1$ and $E C 2$, evaluated from the data, are questionable, making comparison of the levels observed in GaAs:Si:Er and GaAs:Se:Er difficult. Levels $E D 2$ and $E C 2$ have similar emission energies and capture cross sections and are attributed to $E L 2 .{ }^{17}$ Since $E C 1$ appears only in GaAs:Se:Er, it is attributed to the presence of erbium. The peaks detected in GaAs:Se:Er also exhibit a dependence on the electric field applied to the sample. This effect cannot be evaluated as was done for the GaAs:Si:Er sample due to low reverse bias breakdown voltages. In both erbium codoped samples, the introduction of erbium reduces the magnitude of the $E L 2$-related peak. The reduction of the EL2-related peak in both codoped samples indicates that there is interaction of erbium with excess arsenic in the crystal.

\section{DISCUSSION}

In contrast to previous studies, the use of the $\left(t-\mathrm{Bu}_{2} \mathrm{Pz}\right)_{3}(4-t-\mathrm{BuPy})_{2} \mathrm{Er}$ source leads to greatly enhanced 
erbium-related emission at $\sim 1.54 \mu \mathrm{m}$ over the conventional $\mathrm{Cp}-\mathrm{Er}$ sources for similar growth conditions. The improved emission characteristics also occur at a lower total erbium concentration in the present study indicating that this erbium source has improved efficacy over previous source compounds. In this study, the erbium incorporation leads to a variety of electrical and physical changes in GaAs. The surface morphology roughens and there is an introduction of addition levels within the band gap of the material. The appearance of strong and systematic carrier compensation in the GaAs:Er samples indicates that while the erbium concentration may be low, as indicated by the SIMS measurement, there is sufficient introduction of other impurities, notably oxygen and carbon, to lead to appreciable compensation of the intentional shallow dopants. In a previous study of GaAs:Er, the oxygen concentration follows the erbium concentration and is of the same order of magnitude as the erbium concentration. ${ }^{10}$ The fact that the erbium concentration is much less than the oxygen or carbon concentrations suggest that the doping mechanism using the present erbium source is different from that of previously studied Cp-based sources. Under the temperature and V/III ratio conditions used to synthesize samples for this study, the oxygen and carbon concentrations in GaAs grown without the incorporation of erbium would be below the SIMS detection limit. This suggests that the oxygen and carbon levels observed are related to the presence of the erbium source compound during growth. The observed increase in compensation with erbium mole fraction in the reactor is most likely due to the ancillary incorporation of oxygen and carbon, known to be present in our samples, or perhaps other unidentified impurities. This observed compensation is related to introduction of the erbium source into the reactor. Trends in the erbium incorporation efficiency will therefore be assumed to follow the trends in compensation, however the erbium concentration alone cannot account for the observed compensation.

The low erbium concentration incorporated into GaAs at a given $\mathrm{H}_{2}$ flow rate through the bubbler could be due to several issues. The greatest uncertainty in determining the erbium incorporation efficiency is the calculation of the precursor mole fraction in the gas phase. It is difficult to determine an exact value for the mole fraction of the precursor in the reactor ambient due to the uncertainty in estimates for the source vapor pressure. Given the similarity in sublimation temperatures at pressures less than 0.1 Torr for the $\left(t-\mathrm{Bu}_{2} \mathrm{Pz}\right)_{3}(4-t-\mathrm{BuPy})_{2} \mathrm{Er}$ and $\mathrm{Cp}$-based sources, a reasonable assumption is that the enthalpies of sublimation are similar. This assumption yields an estimate for the erbium precursor mole fraction in the reactor with an upper limit of $1 \times 10^{-6}$, which is comparable to values in previous studies using $\mathrm{Cp}$-based precursors. Based on these assumptions, the erbium incorporation efficiency is less than previously investigated in Cp-based Er sources. ${ }^{10,11}$

Parasitic gas phase reactions in the hydrogen-rich reactor ambient could remove the erbium precursor before it can be incorporated into the growing crystal. The decrease in erbium incorporation at increased $\mathrm{V} / \mathrm{III}$ ratios indicates the presence of an arsine-related gas phase or surface reactions. $\mathrm{AsH}_{x}$ species $(x=1-3)$, known to exist in the MOVPE
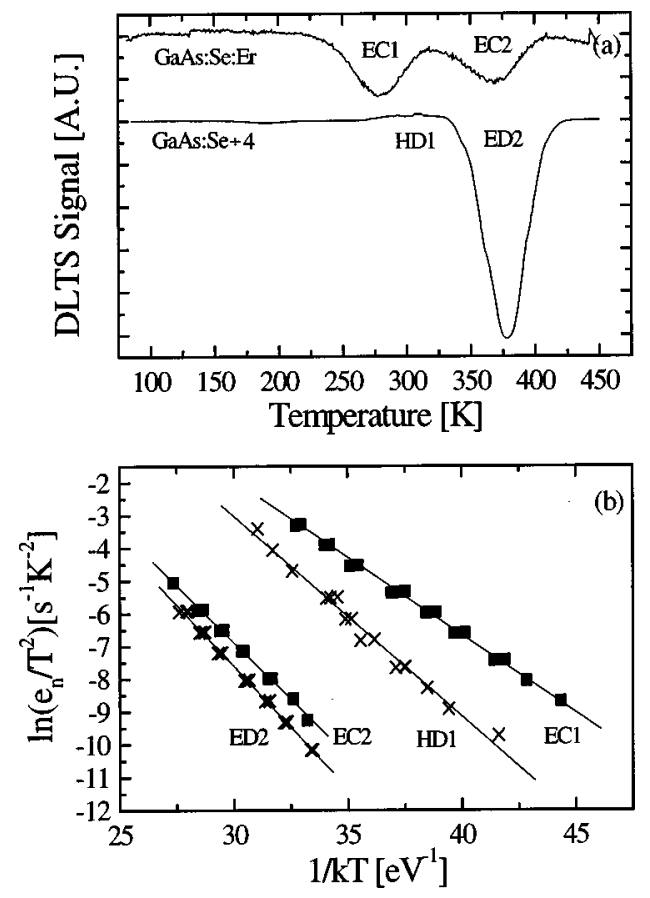

FIG. 5. (a) DLTS spectra for GaAs:Se and GaAs:Se:Er. (b) Arrhenius plots for the levels detected in GaAs:Se $(\times)$ and GaAs:Se:Er (匹). EC2 and ED2 are attributed to the $\mathrm{As}_{\mathrm{Ga}}(E L 2)$ defect (after Ref. 17). EC1 is attributed to erbium incorporation. The origin of $H D 1$ is not known, but it may be related to selenium.

growth environment, could react readily with the erbium precursor to form reaction products that remove erbium from the gas phase. Similar strong effects of $\mathrm{AsH}_{3}$ partial pressure were seen in the growth of GaAs:Er using certain substituted Cp-erbium compounds. ${ }^{10}$ GaAs:Er growth studies using alternative arsenic sources, such as $t$-butylarsine or trimethylarsine, would help determine the specific effect of $\mathrm{AsH}_{x}$ species on erbium incorporation. The observed compensation is likely due to the incorporation of both unintentional carbon, as a shallow acceptor, and oxygen, as a midgap state. Oxygen is known to be coincorporated with erbium and carbon has been reported to be incorporated when Cp-based erbium sources are used to dope GaAs. ${ }^{11}$ Higher growth temperatures and V/III ratios within the reactor generally reduce both the rate of unintentional carbon and oxygen incorporation, which would explain the reduced compensation observed under these growth conditions. ${ }^{21}$

The $\left(t-\mathrm{Bu}_{2} \mathrm{Pz}\right)_{3}(4-t-\mathrm{BuPy})_{2} \mathrm{Er}$ source leads to a center identified as Er-2O (Refs. 15 and 22) in the GaAs film under our particular growth conditions. The erbium optical activity, attributed to this $\mathrm{Er}-2 \mathrm{O}$ center, was found to be higher compared to centers found in GaAs films prepared with other erbium precursors under similar conditions. The identification of this single center does not exclude the presence of other optically active centers, which are not activated by excitation above the host crystal band gap. ${ }^{23}$ The high intensity as well as the narrow line shape to the Er-2O PL emission found in these samples at a low concentration of Er, when compared to previous studies using $\mathrm{Cp}-\mathrm{Er}$ compounds, indicate that the $\mathrm{Er}-2 \mathrm{O}$ is preferentially formed when using this source. Cp-based erbium sources introduce several impurity 
centers leading to broad luminescence features, unless intentional oxygen is added to the reactor during growth. In the present study, sufficient unintentional oxygen was present in the growth ambient to be coincorporated with the erbium. This unintentional oxygen in the present study may come from several sources. $\mathrm{H}_{2} \mathrm{O}$ and tetrahydrofuran may be contaminants introduced into the erbium source during source synthesis. Both molecules, however, would react with pyrazolato ligands to produce a compound which is much less volatile than $\left(t-\mathrm{Bu}_{2} \mathrm{Pz}\right)_{3}(4-t-\mathrm{BuPy})_{2} \mathrm{Er}^{24}$ The most likely sources of oxygen are therefore residual contaminants in the source gases or $\mathrm{H}_{2} \mathrm{O}$ adsorbed on the inner reactor walls during sample loading. Metalorganic source materials, such as TMGa, can have trace amounts of metal alkoxides that lead to oxygen incorporation. ${ }^{25}$

The addition of shallow impurities to GaAs:Er has a strong influence on the $1.54 \mu \mathrm{m}$ luminescence as reported in Fig. 1. Quenching of the erbium-related luminescence by shallow donors has previously been reported for material grown by MBE. In that case, the quenching was attributed to the formation of $\mathrm{Er}-\mathrm{Si}$ moieties. ${ }^{4}$ Based on this present study, such complex formation is unlikely since both Se and $\mathrm{Si}$ codoping results in the same quenching behavior. Si and Se reside on different sublattices and should result in the formation of very different defect complexes. We believe that such erbium-donor complexes do not, therefore, play a role in the quenching behavior seen in our samples.

The quenching of the erbium-related luminescence can be due to the presence of alternative pathways for energy transfer that compete with the pathway leading to excited erbium ions and subsequent emission. An existing model for the excitation of the erbium center involves the formation of a bound exciton on the $\mathrm{Er}^{3+}$ ion that recombines to create an excited $\mathrm{Er}^{3+}$ ion. ${ }^{12} \mathrm{~A}$ high electron and hole concentration would reduce the radiative lifetime for competing band-toband recombination. Such rapid band-to-band recombination would decrease the erbium-related emission in both $n$ - and $p$-type samples. The quenching of erbium-related emission in $n$-type material, but not $p$-type material, does not support this mechanism as the sole competing recombination pathway. Auger energy transfer involving the erbium-bound exciton or the excited $\mathrm{Er}^{3+}$ ion and a band-state electron can also lead to a decrease in erbium-related emission. ${ }^{26}$ An increased electron concentration would lead to an increase in nonradiative Auger recombination. The Auger recombination rate for $n$-type GaAs:Er is known to be much higher than the rate for $p$-type GaAs:Er. ${ }^{27}$ This quenching mechanism has been investigated for the samples in this study with results indicating that this effect is dominated by dissociation of the bound exciton and by promotion of a free electron into the conduction band. ${ }^{28}$

Other defect complexes could also form in competition with the formation of the $\mathrm{Er}-2 \mathrm{O}$ center and lead to a change in the erbium-related emission. Erbium can occupy a displaced tetrahedral interstitial position in GaAs. ${ }^{29}$ Ytterbium in GaAs also occupies a nonsubstitutional site forming an electron trap with an energy of $0.5-0.7 \mathrm{eV}$ below the conduction band. ${ }^{30}$ Seghier et al. performed photoinduced current transient spectroscopy (PICTS) on GaAs doped with erbium or ytterbium. Both elements introduced deep levels at 0.67 and $0.65 \mathrm{eV}$ below the conduction band, respectively. ${ }^{31}$ These reports lead us to assign the EA3 level detected in GaAs:Si:Er as due to nonsubstitutional erbium. The difference between the energy determined in this article and the values from the literature cited can be attributed to the electric field dependence associated with this trap.

\section{v. CONCLUSIONS}

We have investigated a new source, tris(3,5-di- $t$ butylpyrazolato)bis(4-t-butylpyridine)erbium, for the incorporation of erbium into GaAs. Erbium is believed to incorporate in the form of an $\mathrm{Er}-2 \mathrm{O}$ center, as evidenced by the sharp luminescence spectrum characteristic of this specific defect. GaAs doped using the $\left(t-\mathrm{Bu}_{2} \mathrm{Pz}\right)_{3}(4-t-\mathrm{BuPy})_{2} \mathrm{Er}$ source leads to sharper and more intense emission at $\sim 1.54$ $\mu \mathrm{m}$ than samples doped to even higher erbium concentrations using the $\mathrm{Cp}$-based erbium sources. There is a dramatic effect of the sample carrier type on this erbium-related luminescence. The dependence of the erbium-related luminescence on the carrier type is proposed to be due to freeelectron enhanced Auger recombination. DLTS performed on GaAs:Si:Er and GaAs:Se:Er showed several deep levels in the upper half of the conduction band. One of these states was identified as being related to the incorporation of erbium into GaAs. This defect is proposed to be erbium occupying a nonsubstitutional lattice site.

${ }^{1}$ G. Franzo, F. Priolo, C. Spinella, and S. Coffa, J. Appl. Phys. 81, 2784 (1997).

${ }^{2}$ J. Michel, B. Zheng, J. Palm, E. Ouellette, F. Gan, and L. C. Kimerling, Mater. Res. Soc. Symp. Proc. 422, 317 (1996).

${ }^{3}$ P. N. Favennec, H. L'Haridon, M. Salvi, D. Moutonnet, and Y. Le Guillou, Electron. Lett. 25, 218 (1989).

${ }^{4}$ K. R. Evans, E. N. Taylor, C. E. Stutz, D. W. Elsaesser, J. E. Colon, Y. K. Yeo, R. L. Hengehold, and J. S. Solomon, J. Vac. Sci. Technol. B 10, 870 (1992).

${ }^{5}$ P. Rutter, K. E. Singer, A. R. Peaker, and A. C. Wright, Mater. Res. Soc. Symp. Proc. 422, 35 (1996).

${ }^{6}$ J. E. Colon, D. W. Elsaesser, Y. K. Yeo, R. L. Hengehold, and G. S. Pomrenke, Mater. Res. Soc. Symp. Proc. 301, 169 (1993).

${ }^{7}$ F. Bantien, E. Bauser, and J. Weber, J. Appl. Phys. 61, 2803 (1987).

${ }^{8}$ K. Uwai, H. Nakagome, and K. Takahei, J. Cryst. Growth 93, 583 (1988).

${ }^{9}$ C. R. Lewis, W. T. Dietze, and M. J. Ludowise, J. Electron. Mater. 12, 507 (1983).

${ }^{10}$ J. M. Redwing, T. F. Kuech, D. C. Gordon, B. A. Vaartstra, and S. S. Lau, J. Appl. Phys. 76, 1585 (1994).

${ }^{11}$ K. Takahei and A. Taguchi, J. Appl. Phys. 74, 1979 (1993).

${ }^{12}$ T. D. Culp, U. Hömmerich, J. M. Redwing, T. F. Kuech, and K. L. Bray, J. Appl. Phys. 82, 368 (1997).

${ }^{13}$ A. C. Greenwald, K. J. Linden, W. S. Rees, Jr., O. Just, N. M. Haegel, and S. Donder, Mater. Res. Soc. Symp. Proc. 422, 63 (1996).

${ }^{14}$ D. Pfeiffer, L. M. Liable-Sands, A. L. Rheingold, M. J. Heeg, and C. H. Winter (unpublished).

${ }^{15}$ K. Takahei, R. A. Hogg, and A. Taguchi, Mater. Res. Soc. Symp. Proc. 422, 267 (1996).

${ }^{16}$ L. Samuelson, P. Omling, H. Titze, and H. G. Grimmeiss, J. Cryst. Growth 55, 164 (1981).

${ }^{17}$ G. Martin and S. Makram-Ebeid, in Deep Centers in Semiconductors: A State-of-the-Art Approach, 2nd ed., edited by S. T. Pantelides (Gordon and Breach Science, Philadelphia, 1992), p. 401.

${ }^{18} \mathrm{~J}$. Bourgoin and M. Lannoo, Point Defects in Semiconductors II (Springer, Berlin, 1983), pp. 199-201.

${ }^{19}$ J. Frenkel, Phys. Rev. 54, 647 (1938). 
${ }^{20}$ Z. C. Huang, B. Yang, H. K. Chen, and J. C. Chen, Appl. Phys. Lett. 66, 2745 (1995).

${ }^{21}$ T. F. Kuech and J. M. Redwing, J. Cryst. Growth 145, 382 (1994).

${ }^{22}$ K. Takahei, Y. Horikoshi, and A. Taguchi, Mater. Sci. Forum 196-201, 639 (1995)

${ }^{23}$ R. A. Hogg, K. Takahei, and A. Taguchi, J. Appl. Phys. 79, 8682 (1996).

${ }^{24} \mathrm{C}$. H. Winter (private communication).

${ }^{25}$ J. G. Cederberg, K. L. Bray, and T. F. Kuech, J. Appl. Phys. 82, 2263 (1997).

${ }^{26}$ T. Benyattou, D. Seghier, G. Guillot, R. Moncorge, P. Galtier, and M. N. Charasse, Appl. Phys. Lett. 58, 2132 (1991).
${ }^{27}$ P. T. Landsberg, Recombination in Semiconductors (Cambridge University Press, Cambridge, 1991), pp. 369-446.

${ }^{28}$ T. D. Culp, J. G. Cederberg, B. Bieg, T. F. Kuech, K. L. Bray, D. Pfeiffer, and C. H. Winter, J. Appl. Phys. 83, 4918 (1998).

${ }^{29}$ K. Takahei, A. Taguchi, Y. Horikoshi, and J. Nakata, J. Appl. Phys. 76, 4332 (1994)

${ }^{30}$ A. Taguchi, H. Nakagome, and K. Takahei, J. Appl. Phys. 68, 3390 (1990).

${ }^{31}$ D. Seghier, T. Benyattou, A. Kalboussi, S. Moneger, G. Marrakchi, G. Guillot, B. Lambert, and A. Guivarc'h, J. Appl. Phys. 75, 4171 (1994). 